\title{
Finite Element Analysis of Room Temperature Nanoimprint Lithography Process with Rate Dependent Plasticity
}

\author{
J. H. Song ${ }^{1, a}$, H. Huh ${ }^{1, b}$, S. H. Kim ${ }^{2, c}$ and H. T. Hahn ${ }^{3, d}$ \\ ${ }^{1}$ Department of Mechanical Engineering, Korea Advanced Institute of Science and Technology \\ Science Town, Daejeon, 305-701, Korea. \\ ${ }^{2} \mathrm{MTI}$ Group, Samsung Corning Precision Glass Co., Ltd., \\ Tangjun-Myun, Asan-Si, Chungnam, 336-840, Korea \\ ${ }^{3}$ Mechanical \& Aerospace Engineering Department, University of California, Los Angeles \\ Los Angeles, CA 90095-1597, U.S.A. \\ ajhsong_me@kaist.ac.kr, ${ }^{b}$ hhuh@kaist.ac.kr, 'seung-ho.kim@samsung.com, ${ }^{\text {dhahna@ } @ s e a s . u c l a . e d u}$
}

Keywords: room temperature nanoimprint lithography, rate dependent plasticity, finite element method, process parameter.

\begin{abstract}
Nanoimprint lithography (NIL) process at the room temperature has been proposed newly to achieve the shape accuracy and to overcome the sticking problem induced in conventional NIL processes. Success of the room temperature NIL relies on the complete understanding of the mechanical behavior of the polymer. Since a conventional NIL process has to heat a polymer above the glass transition temperature to deform the physical shape of the polymer with a mold pattern, visco-elastic properties of the polymer have major effect on the NIL process. The rate dependent behavior of the polymer is also important in the room temperature NIL process because a mold is rapidly pressed onto the polymer while there has been no study on the rate-dependent NIL process. In this paper, finite element analysis of the room temperature NIL process is performed with the consideration of the strain-rate dependent behavior of the polymer. The analyses with the variation of the imprinting speed and the imprinting pattern are carried out in order to investigate the effect of the process parameters on the room temperature NIL process. The analysis results show that the deformed shape and the imprinting force are diversified with the variation of the imprinting speed due to the dynamic behavior of the polymer with the rate dependent plasticity model. The results provide a guideline to determine the process conditions in the room temperature NIL process.
\end{abstract}

\section{Introduction}

Nanoimprint lithography (NIL) has attracted broad interest as a low-cost method to fabricate nanometer-scale patterns in recent years [1,2]. A conventional NIL process must heat the polymer above its grass transition temperature to deform the physical shape of the polymer with the mold and then cool down after fabrication. A conventional NIL process, thus, requires a thermal cycle of the polymer. This heating process causes serious problems in the pattern accuracy and productivity due to the thermal cycle of the polymer. Another problem is the partial removal or peeling-off of the resist during separation of the sample and the mold. A room temperature NIL has been newly proposed to overcome those problems $[3,4]$. The room temperature NIL is faster and more accurate than the conventional NIL because it does not require heating and cooling steps. Success of the room temperature nanoimprint lithography relies on the adequate conditions of the process parameters such as the pressure, the speed, and the shape of mold as well as the mechanical behavior of the polymer such as the yield strength and elongation. It is well known that the strain-rate hardening of a polymer is remarkable and inherent behavior at room temperature. Due to this reason, it is indispensable for the success of the process to consider the rate dependent behavior of the polymer in the room temperature NIL process. 


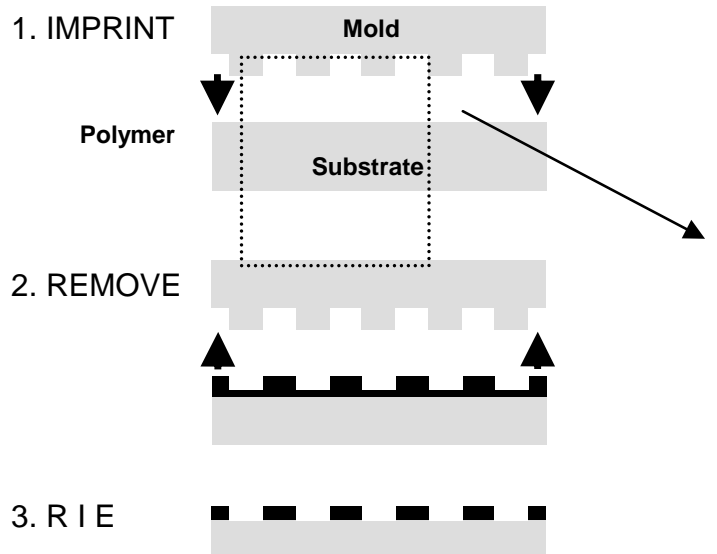

(a) room temperature NIL process

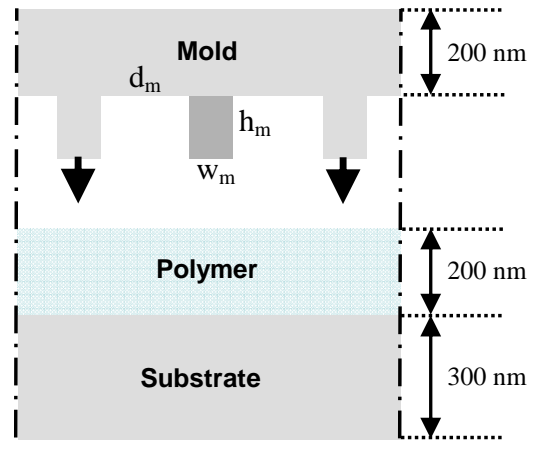

(b) FE analysis model

Fig. 1. Schematic description and analysis model of the room temperature NIL process:

Numerical simulation of manufacturing processes is an essential element in the integration of computer aided engineering. It provides preliminary information for the design of tooling and determination of process conditions. Computer aided engineering with finite element simulation has been recently extended into the conventional NIL process. Young [5] performed the finite element analysis with a viscous model of the polymer. Hirai et al. [6] evaluated the deformation behavior of the polymer with hyper-elastic model with respect to the mold shape and compared the numerical results with the experimental ones. Hirai et al. [7] and Colburn et al. [8] investigated the thermal cycles in heating and cooling steps. Reu et al. [9] studied the mark distortion with the help of finite element method. Weisbrod et al. [10] analyzed the thermal stress in the conventional NIL.

In this paper, finite element analysis of the room temperature NIL process is carried out considering the strain rate dependent behavior of the polymer. The Johnson-Cook model is utilized in the analysis for the description of the strain-rate hardening phenomenon [11,12]. The deformed shape and the imprinting force are evaluated with the variation of imprinting speed and the imprinting pattern in order to investigate the effect of the process parameters on the room temperature NIL process. The analysis results show that the deformed shape and the imprinting force are very much diversified with the variation of the imprinting speed because the dynamic behavior of the polymer is remarkably changed with the deformation rate.

\section{Finite element simulation of nanoimprint lithography process}

Finite element analysis model. The room temperature NIL process is illustrated in Fig.1 (a). First, the patterned mold is pressed onto a substrate coated with the polymer. Then, the mold is separated from the substrate, leaving the fabricated patterns on the substrate. Lately, reactive ion etching (RIE) step is followed. In the analysis, only the imprint step is considered in the present study because the deformed profile of the polymer is principally determined in the imprint step. Although tens of repeated nanopatterns are defined on the mold, it is impractical to consider all nanopatterns in the finite element analysis with the drastic analysis time. A simplified model shown in Fig. 1 (b) is selected and it is regarded as the unit cell of the pattered mold by taking repeated symmetric boundary conditions into account. The depth of mold groove, $h_{m}$, and the width of mold, $w_{m}$, is set to be $120 \mathrm{~nm}$ and $50 \mathrm{~nm}$ respectively. The distance between each nanopattern, $d_{m}$, is assumed to be $200 \mathrm{~nm}$. The angular corner of the mold groove is rounded to the radius of $5 \mathrm{~nm}$ in order to avoid the stress concentration of the polymer. The coulomb friction coefficient between the mold and polymer is assumed to be 0.2 . The polymer used in the analysis is the polypropylene. The plane strain elements are used for the polymer and rigid elements for the mold and substrate. The analysis employs a commercial explicit finite element code, LS-DYNA 3D [13]. 


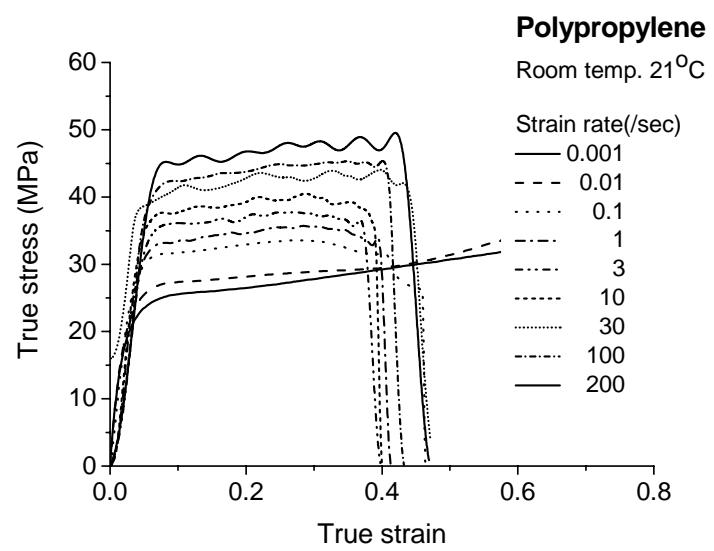

(a) true stress-strain curve

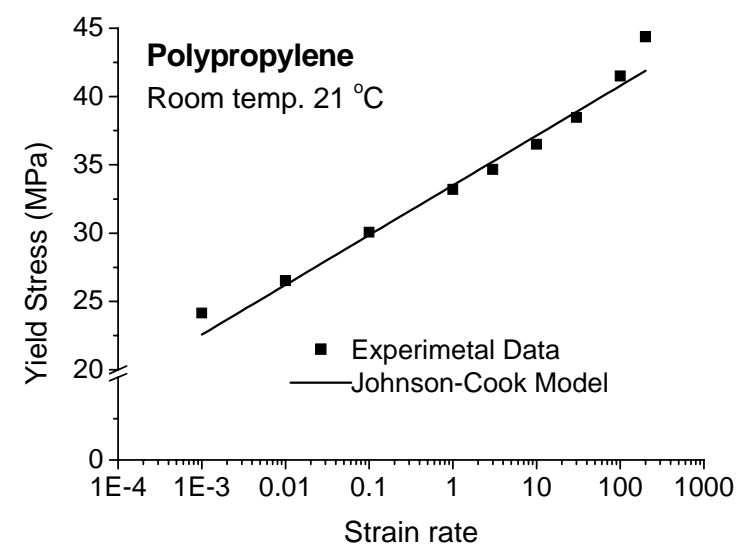

(b) strain rate sensitivity

Fig. 2. Strain rate dependent behavior of the polypropylene.

Strain-rate dependent behavior of polymer. The strain rate hardening of a polymer is so remarkable at the room temperature that the rate dependent plasticity model is indispensably utilized in the nanoimprint analysis. Fig.2 (a) shows the stress-strain curves of the polypropylene with different strain rates obtained from the experiment using the high speed tensile test machine [14]. Fig. 2 (b) explains the strain rate sensitivity of the polypropylene considering the yield stress. The strainrate sensitivity shown in Fig. 2 (b) is interpolated with the Johnson-Cook model represented by Eq. 1 and imposed on the finite element analysis of the nanoimprint process.

$$
\bar{\sigma}=\left(A+B \bar{\varepsilon}^{n}\right)(1+C \ln \overline{\dot{\varepsilon}})\left(1-T^{* m}\right)
$$

where $T^{*}$ is the homogeneous temperature represented by Eq. 2.

$$
T^{*}=\frac{T-T_{\text {room }}}{T_{\text {melt }}-T_{\text {room }}}
$$

Results of the unit model. Simulation of the imprint process is carried out under a constant imprinting speed of $20 \mathrm{~nm} / \mathrm{sec}$. The total stroke of mold is set to be same as the depth of the patterned mold, $h_{m}$. The deformed patterns of the imprinted polymer are shown in Fig. 3 for different imprinting strokes. At first two stages of $30 \mathrm{~nm}$ and $60 \mathrm{~nm}$ imprinting stroke, the polymer near the patterned mold is deformed downward due to the imprint feature and the polymer between the patterned mold

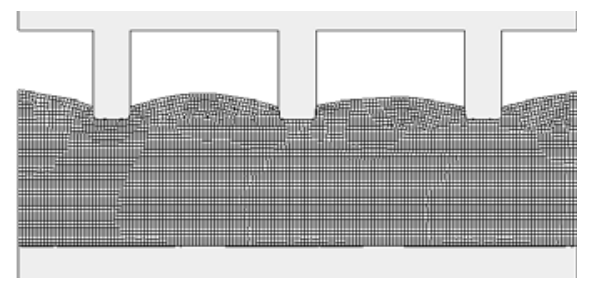

(a) stroke $30 \mathrm{~mm}$

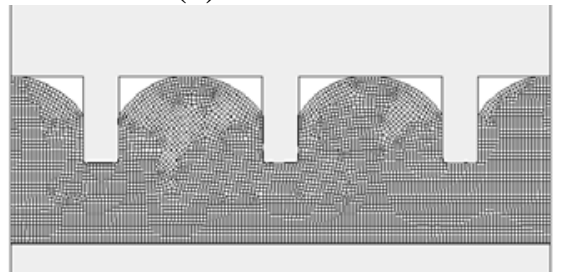

(c) stroke $90 \mathrm{~mm}$

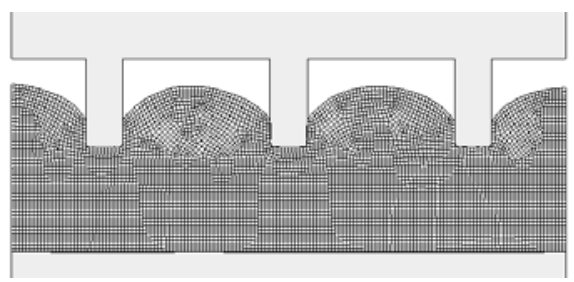

(b) stroke $60 \mathrm{~mm}$

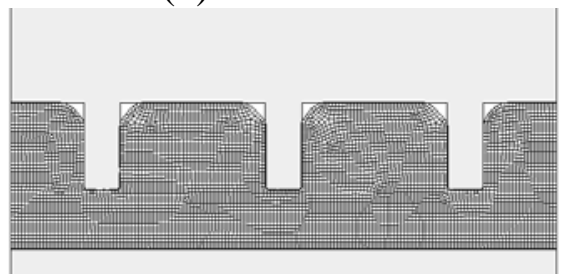

(d) stroke $120 \mathrm{~mm}$

Fig. 3. Deformed shapes of the polymer with the constant imprinting speed of $20 \mathrm{~nm} / \mathrm{sec}$. 


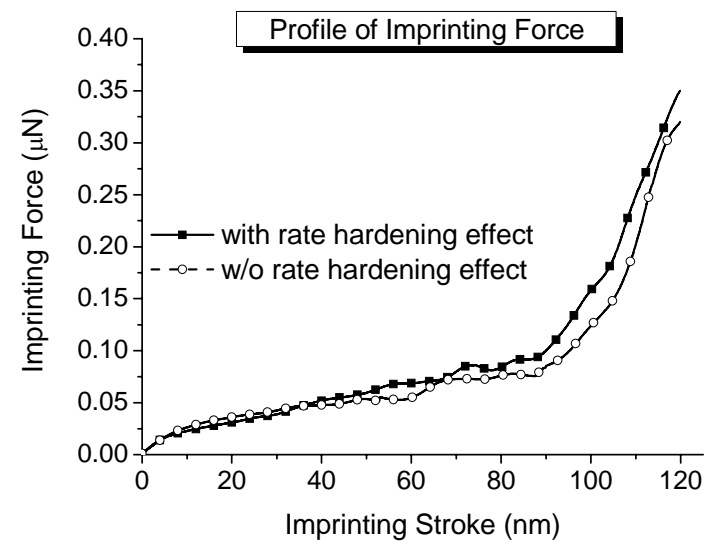

(a) imprinting force

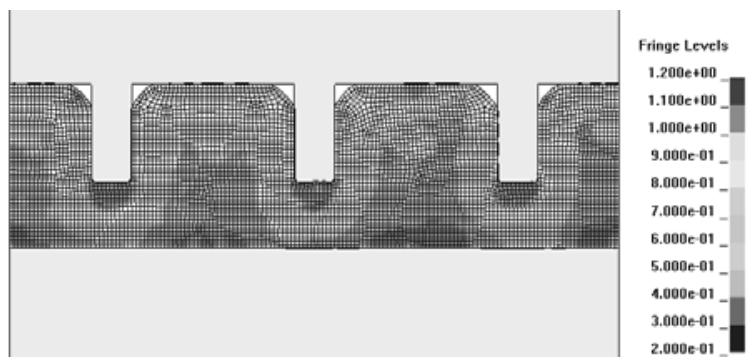

(b) the effective plastic strain

Fig. 4. Profile of the imprinting force and the effective strain with imprinting speed of $20 \mathrm{~nm} / \mathrm{sec}$.

is relatively deformed upward because the imprint feature pushes nearby material outward. At the 90 $\mathrm{nm}$ imprinting stroke, the base of mold starts to compress the polymer film. Further compression causes the polymer material to spread out and fill the corner area.

The variation of the imprinting force during the imprinting process is shown in Fig. 4 (a). The imprinting force is slowly increased before the base of the mold touch the polymer film. As the mold base starts to compress the polymer film at the imprinting stroke of $90 \mathrm{~nm}$, the imprinting force distinctly increases due to the increase of compressed area. Fig. 4 (a) also explains that the punch force obtained from the finite element analysis with the Johnson-Cook model is larger than that with the generally used static model. It is because that the Johnson-Cook model enables to represent the strain- rate hardening effect of polymer during the nanoimprint process. Fig. 4(b) shows the distribution of the effective plastic strain. The deformation is highly concentrated near the imprinting feature of the mold.

\section{Effect of process parameters}

Imprinting speed. Finite element analysis with different imprinting speeds from $20 \mathrm{~nm} / \mathrm{s}$ to 500 $\mathrm{nm} / \mathrm{s}$ is performed in order to evaluate the effect of rate dependency accurately during the nanoimprint process. The deformed patterns of the imprinted polymer with a constant imprinting speed of $500 \mathrm{~nm} / \mathrm{s}$ are shown in Fig. 5 . The deformed patterns are quite different from the speed of 20 $\mathrm{nm} / \mathrm{s}$ in that the deformation tends to be localized and has been an effect on the neighborhood of the patterns

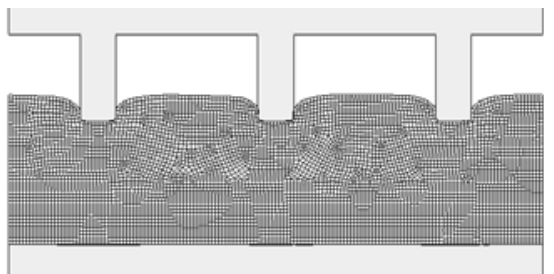

(a) stroke $30 \mathrm{~mm}$

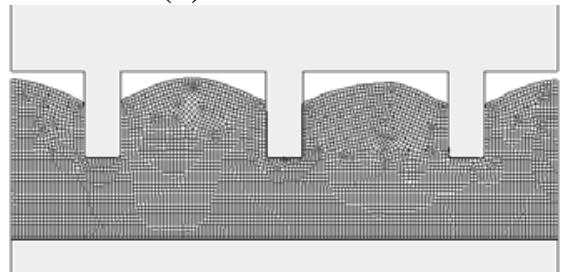

(c) stroke $90 \mathrm{~mm}$

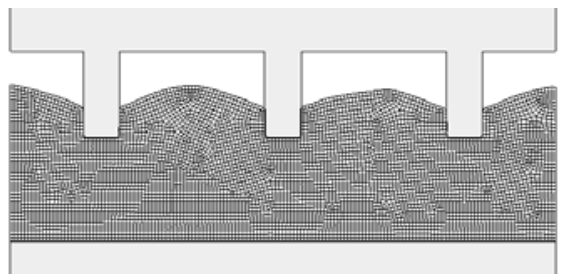

(b) stroke $60 \mathrm{~mm}$

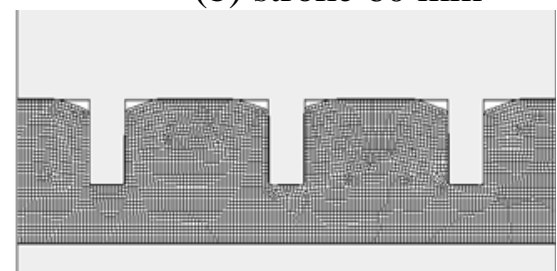

(d) stroke $120 \mathrm{~mm}$

Fig. 5. Deformed shape of the polymer with the constant imprinting speed of $500 \mathrm{~nm} / \mathrm{sec}$ 


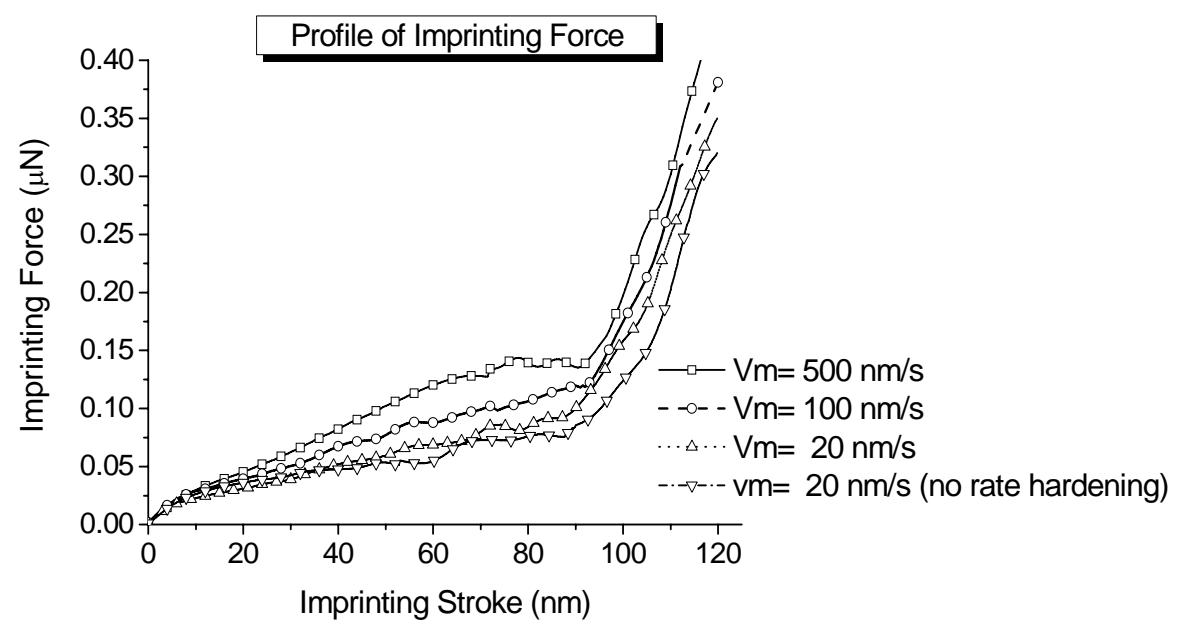

Fig. 6. Variation of the imprinting force with respect to the imprinting speed.

initially at the imprint stroke of $30 \mathrm{~nm}$. In the condition of the imprinting speed of $20 \mathrm{~nm} / \mathrm{s}$, the base of mold starts to compress the polymer film at the stroke of $90 \mathrm{~nm}$. However, the mold and polymer do not contacted each other at the stroke of $90 \mathrm{~nm}$ in the imprinting speed of $500 \mathrm{~nm} / \mathrm{s}$ as shown in Fig. 5 (c), Fig. 6 depicts the imprinting force with the variation of the imprinting speed. Due to the strainrate hardening of the polymer, the imprinting force is increased as the imprinting speed increases. The results demonstrate that it is necessary to consider the strain rate hardening effect in the simulation of room temperature NIL process.

Imprinting pattern. In order to examine the effect of the imprinting nanopattern on the deformed shape and the imprinting force, finite element analysis is carried out with different height of nano pattern and distance between nanopatterns. The height of nanopattern, $\mathrm{h}_{\mathrm{m}}$, is set to be varied from 80 $\mathrm{nm}$ to $160 \mathrm{~nm}$ and the distance between nanopatterns, $\mathrm{d}_{\mathrm{m}}$, is from $100 \mathrm{~nm}$ to $300 \mathrm{~nm}$. Other dimensions are same as the unit model shown in Fig. 1. The imprinting speed is set to be $20 \mathrm{~nm} / \mathrm{s}$. The deformed shapes of the polymer at the final state with respect to the imprinting nanopatterns are shown in Fig. 7. The figure illustrates that the distance of each nanopattern has more influence on the filling amount of the imprinted pattern rather than the aspect ratio of the imprinting pattern. It is because that the small distance enables the polymer material to flow outward easily and fill the corner area. Fig. 8 shows the profiles of the imprinting force with different imprinting nanopatterns. The figure represents that the height of the imprinting nanopattern highly has a great effect on the maximum imprinting force.

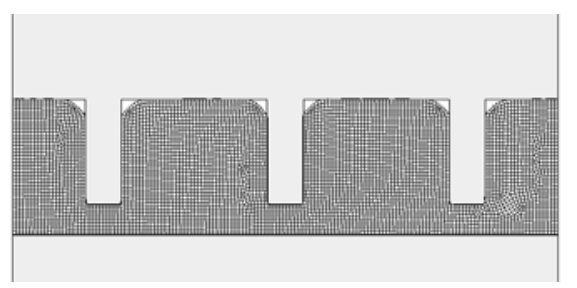

(a) $h_{m}=160 \mathrm{~mm}$

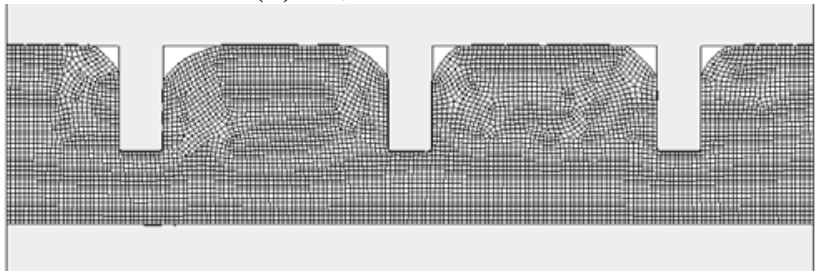

(c) $d_{m}=300 \mathrm{~mm}$

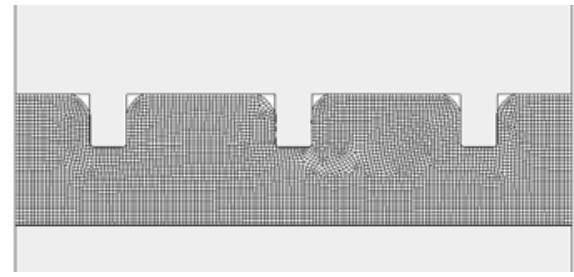

(b) $h_{m}=80 \mathrm{~mm}$

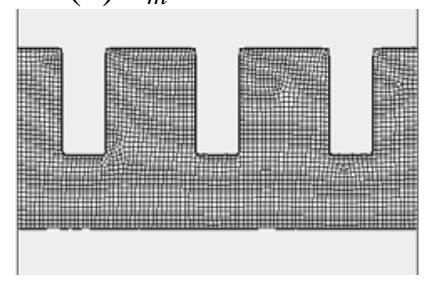

(d) $d_{m}=100 \mathrm{~mm}$

Fig. 7. Deformed shape of the polymer with respect to the imprinting nanopatterns. 


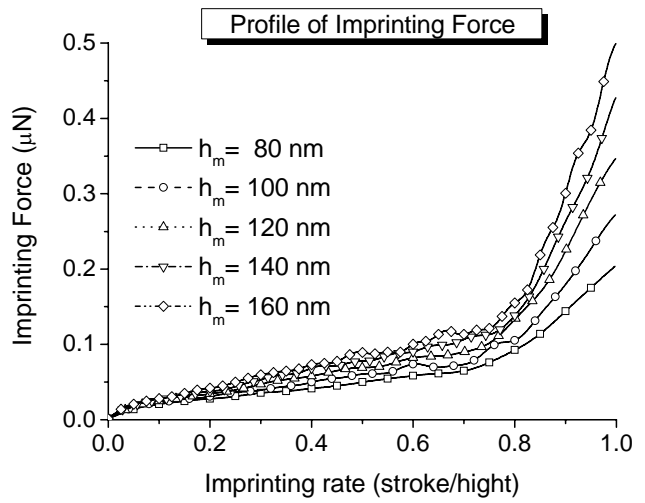

(a) height of nanopattern

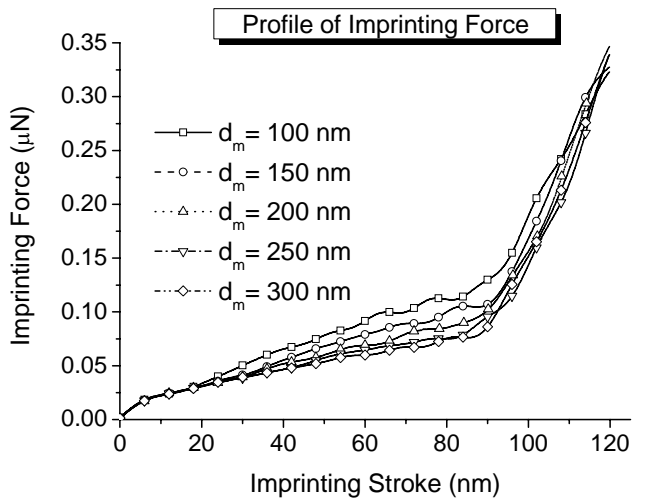

(b) distance between nanopatterns

Fig. 8. Variation of the imprinting force with respect to the imprinting nanopatterns.

\section{Summary}

This paper is concerned with the finite element analysis of the room temperature NIL with the consideration of the rate-dependent behavior of the polymer. The analyses with the variation of the imprinting speed and the imprinting pattern are carried out in order to investigate the effect of the two process parameters. The analysis results show that the deforming shape in the process is remarkably changed with respect to the imprinting speed because of the dynamic behavior of the polymer. The imprinting force is increased with the increase of the imprinting speed as well. The results also indicate that the distance of the nanopattern has significant influence on the rectangularity of the imprinted pattern and the depth of the imprinting nanopattern has a great effect in the imprinting force. Tel: +82-42-869-3262, Fax: +82-42-869-3210, e-mail: jhsong_me@kaist.ac.kr

\section{References}

[1] S. Y. Chou, P. R. Krauss and P. J. Renstrom: Science Vol. 272 (1996), p. 85.

[2] X. Sun, L. Zhuang, W. Zhang and S. Y. Chou: J. Vac. Sci. Technol. B Vol. 16 (1998), p. 3922.

[3] P. S. Hong and H. H Lee: Appl. Phys. Lett. Vol. 83 (2003), p. 2441.

[4] S. Matsui, Y. Igaku, H. Ishigaki, J. Fujita, M. Ishida, Y. Ochiai, H. Namatsu and M. Komuro: J. Vac. Sci. Technol. B Vol. 21 (2003), p. 688.

[5] W. -B. Young: Microelectron. Eng. Vol. 77 (2005), p. 405

[6] Y. Hirai, S. Yoshida, A. Okamoto, Y. Tanaka, M. Endo, S. Irie, H. Nakagawa and M. Sasago: J. Vac. Sci. Technol. B Vol. 19 (2001), p. 2811.

[7] Y. Hirai, S. Yoshida and N. Takagi: J. Vac. Sci. Technol. B Vol. 21 (2003), p. 2765.

[8] M. Colburn, I. Seuz, B. J. Choi, M. Meissl, T. Bailey, S. V. Sreenivasan, J. G. Ekerdt and C. G. Willson: J. Vac. Sci. Technol. B Vol. 19 (2001), p. 2685.

[9] P. L. Reu, R. L. Engelstad and E. G. Lovell: Microelectron. Eng. Vol. 69 (2003), p. 420.

[10] E. J. Weisbrod: J. Vac. Sci. Technol. B Vol. 20 (2002), p. 3047.

[11] G. R. Johnson and W. H. Cook, Proc. 7th International Symposium on Ballistics (1983) p. 541.

[12] W. J. Kang and H. Huh: Exp. Mech. Vol. 42, (2002), p. 8.

[13] J. O. Hallquist: LS-DYNA 3D Keyword Manual. (LSTC, USA, 1998).

[14] J. H. Lim and H. Huh: submitted to Exp. Mech. (2005) 